\title{
Generalized $\beta$-conformal change and special Finsler spaces
}

\author{
Nabil L. Youssef ${ }^{\dagger}$, S. H. Abed ${ }^{\ddagger}$ and S. G. Elgendi ${ }^{\sharp}$ \\ ${ }^{\dagger}$ Department of Mathematics, Faculty of Science, \\ Cairo University, Giza, Egypt \\ nlyoussef2003@yahoo.fr, nyoussef@frcu.eun.eg \\ ${ }^{\ddagger}$ Department of Mathematics, Faculty of Science, \\ Cairo University, Giza, Egypt \\ sabed52@yahoo.fr, sabed@frcu.eun.eg \\ \#Department of Mathematics, Faculty of Science, \\ Benha University, Benha, Egypt \\ salahelgendi@yahoo.com
}

\begin{abstract}
In this paper, we investigate the change of Finslr metrics

$$
L(x, y) \longrightarrow \bar{L}(x, y)=f\left(e^{\sigma(x)} L(x, y), \beta(x, y)\right),
$$

which we refer to as a generalized $\beta$-conformal change. Under this change, we study some special Finsler spaces, namely, quasi C-reducible, semi C-reducible, C-reducible, $C_{2}$-like, $S_{3}$-like and $S_{4}$-like Finsler spaces. We obtain some characterizations of the energy $\beta$-change, the Randers change and the Kropina change. We also obtain the transformation of the T-tensor under this change and study some interesting special cases. We then impose a certain condition on the generalized $\beta$-conformal change, which we call the b-condition, and investigate the geometric consequences of such a condition. Finally, we give the conditions under which a generalized $\beta$-conformal change is projective and generalize some known results in the literature.
\end{abstract}

Keywords: Generalized $\beta$-conformal change, $\beta$-conformal change, Randers change, Kropina change, projective change, special Finsler spaces, b-condition, T-tensor. 2000 Mathematics Subject Classification. 53B40, 53B05. 


\section{Introduction}

Throughout, $M$ is an n-dimensional $C^{\infty}$ differentiable manifold and $F^{n}=(M, L)$ a Finsler space equipped with the fundamental function $L(x, y)$ on $T M$.

Finsler geometry was first introduced by Finsler himself, to be studied by many eminent mathematicians for its theoretical importance and applications in the variational calculus, mechanics and theoretical physics. Moreover, the dependence of the fundamental function $L(x, y)$ on both the positional argument $x$ and directional argument $y$ offers the possibility to use it to describe the anisotropic properties of the physical space. For a differential one-form $\beta(x, d x)=b_{i}(x) d x^{i}$ on $M$, Randers [17, in 1941, introduced a special Finsler space with the Finsler change $\bar{L}=L+\beta$, where $L$ is Riemannian, to consider a unified field theory $\left(L=\sqrt{a_{i j} y^{i} y^{j}}, a_{i j}\right.$ being the gravitational tensor field and $b_{i}(x)$ the electromagnetic potential). Masumoto [11], in 1974, studied Randers and generalized Randers changes in which $L$ is Finslerian. Kropina [8] introduced the change $\bar{L}=L^{2} / \beta$, where $L$ is Riemannian. This change has been studied by other authors such as Shibata [18] and Matsumoto [9]. Randers and Kropina changes are closely related to physical theories and so Finsler spaces with these metrics have been investigated by many authors, from various approaches in both the physical and mathematical aspects ([3], [1], [20, [22], 23], [24], [26]). Randers change was also applied to the theory of the electron microscope by R. S. Ingarden [6]. Moreover, there is some relation between the Kropina metric and the Lagrangian function of analytic dynamics [18]. In 1984, Shibata [19] considered the general case of any $\beta$-change, that is, $\bar{L}=f(L, \beta)$, thus generalizing many changes in Finsler geometry ([11], [18]). In this context, he studied some special Finsler spaces, such as C-reducible and $S_{4}$-like, under Randers change.

On the other hand, in 1976, Hashiguchi [4] studied the conformal change of a Finsler metric, namely, $\bar{L}=e^{\sigma(x)} L$. In particular, he also dealt with the special conformal transformation named $\mathrm{C}$-conformal. This change has been studied by Izumi [7] among others. In 2008, Abed ([1], 2]) introduced the change $\bar{L}=e^{\sigma(x)} L+\beta$, which he called a $\beta$-conformal change, thus generalizing the conformal, Randers and generalized Randers changes. Moreover, he studied some special Finsler space under this change such as C-reducible, $S_{3}$-like and $S_{4}$-like.

In [25], the present authors introduced and investigated the more general change of Finsler metrics:

$$
L(x, y) \rightarrow \bar{L}=f\left(e^{\sigma(x)} L(x, y), \beta(x, y)\right)=f(\widetilde{L}, \beta)
$$

where $\widetilde{L}=e^{\sigma(x)} L$ and $f$ is a positively homogeneous function of $\widetilde{L}$ and $\beta$ of degree one. They obtained the difference between Cartan connection associated with $(M, L)$ and Cartan connection associated with $(M, \bar{L})$, also, they established some interesting results and computed the torsion and curvature tensors of the transformed space $(M, \bar{L})$ for the four fundamental connections in Finsler geometry. This change is referred to as a generalized $\beta$-conformal change. It is clear that this change is a generalization of all the above mentioned changes and deals simultaneously with $\beta$ change and conformal change. It combines both cases of Shibata $(\bar{L}=f(L, \beta))$ and that of Hashiguchi $\left(\bar{L}=e^{\sigma} L\right)$.

In this paper, we continue our investigation of the generalized $\beta$-conformal change. Under this change, we study some special Finsler spaces, compute the transformed 
T-tensor, introduce what we call b-condition and study when this change becomes projective.

The present paper is organized as follows. In section 1, we introduce the necessary material and background required for the present work. In section 2 , we deal with some special Finsler spaces under a generalized $\beta$-conformal change, namely, quasi C-reducible, Semi C-reducible, C-reducible, $C_{2}$-like, $S_{3}$-like and $S_{4}$-like. In section 3 , we compute the T-tensor of the transformed space under a generalized $\beta$-conformal change and study some interesting special cases. In section 4 , we impose a certain condition on the generalized $\beta$-conformal change, which we call the b-condition, and investigate the geometric consequences of such a condition. Finally, in section 5, we give the conditions under which a generalized $\beta$-conformal change is projective and generalize some known results in the literature.

\section{Notations and preliminaries}

Throughout the present paper we use the terminology and notations of [25]. Let $(M, L)$ be an n-dimensional $C^{\infty}$ Finsler manifold; L being the fundamental Finsler function. Let $\left(x^{i}\right)$ be the coordinates of any point of the base manifold $\mathrm{M}$ and $\left(y^{i}\right) \mathrm{a}$ supporting element at the same point. We use the following notations:

$\partial_{i}$ : partial differentiation with respect to $x^{i}$,

$\dot{\partial}_{i}$ : partial differentiation with respect to $y^{i}$ (basis vector fields of the vertical bundle), $g_{i j}:=\frac{1}{2} \dot{\partial}_{i} \dot{\partial}_{j} L^{2}=\dot{\partial}_{i} \dot{\partial}_{j} E$ : the Finsler metric tensor; $E:=\frac{1}{2} L^{2}$ : the energy function, $l_{i}:=\dot{\partial}_{i} L=g_{i j} l^{j}=g_{i j} \frac{y^{j}}{L}$ : the normalized supporting element; $l^{i}:=\frac{y^{i}}{L}$,

$l_{i j}:=\dot{\partial}_{i} l_{j}$,

$h_{i j}:=L l_{i j}=g_{i j}-l_{i} l_{j}:$ the angular metric tensor,

$C_{i j k}:=\frac{1}{2} \dot{\partial}_{k} g_{i j}=\frac{1}{4} \dot{\partial}_{i} \dot{\partial}_{j} \dot{\partial}_{k} L^{2}:$ the Cartan tensor,

$G^{i}$ : the components of the canonical spray associated with $(M, L)$,

$N_{j}^{i}:=\dot{\partial}_{j} G^{i}$ : the Barthel (or Cartan nonlinear) connection associated with $(M, L)$,

$\delta_{i}:=\partial_{i}-N_{i}^{r} \dot{\partial}_{r}$ : the basis vector fields of the horizontal bundle,

$G_{j h}^{i}:=\dot{\partial}_{h} N_{j}^{i}=\dot{\partial}_{h} \dot{\partial}_{j} G^{i}$ : the coefficients of Berwald connection,

$C_{j k}^{i}:=g^{r i} C_{r j k}=\frac{1}{2} g^{i r} \dot{\partial}_{k} g_{r j}:$ the $\mathrm{h}(\mathrm{hv})$-torsion tensor,

$\gamma_{j k}^{i}:=\frac{1}{2} g^{i r}\left(\partial_{j} g_{k r}+\partial_{k} g_{j r}-\partial_{r} g_{j k}\right):$ the Christoffel symbols with respect to $\partial_{i}$,

$\Gamma_{j k}^{i}:=\frac{1}{2} g^{i r}\left(\delta_{j} g_{k r}+\delta_{k} g_{j r}-\delta_{r} g_{j k}\right)$ : the Christoffel symbols with respect to $\delta_{i}$,

$\left(\Gamma_{j k}^{i}, N_{j}^{i}, C_{j k}^{i}\right)$ : The Cartan connection $C \Gamma$.

For a Cartan connection $\left(\Gamma_{j k}^{i}, N_{j}^{i}, C_{j k}^{i}\right)$, we define

$X_{j \mid k}^{i}:=\delta_{k} X_{j}^{i}+X_{j}^{m} \Gamma_{m k}^{i}-X_{m}^{i} \Gamma_{j k}^{m}$ : the horizontal covariant derivative of $X_{j}^{i}$,

$\left.X_{j}^{i}\right|_{k}:=\dot{\partial}_{k} X_{j}^{i}+X_{j}^{m} C_{m k}^{i}-X_{m}^{i} C_{j k}^{m}$ : the vertical covariant derivative of $X_{j}^{i}$.

Let $F^{n}=(M, L)$ be an n-dimensional Finsler space with a fundamental function $L=L(x, y)$. Consider the following change of Finsler structures, which will be called a generalized $\beta$-conformal change,

$$
L(x, y) \rightarrow \bar{L}(x, y)=f\left(e^{\sigma(x)} L(x, y), \beta(x, y)\right)=f(\widetilde{L}, \beta),
$$


where $f$ is a positively homogeneous function of degree one in $\widetilde{L}=e^{\sigma} L$ and $\beta$ and $\beta=b_{i}(x) d x^{i}$. Assume that $\bar{F}^{n}=(M, \bar{L})$ has the structure of a Finsler space. Entities related to $\bar{F}^{n}$ will be denoted by barred symbols.

We define

$$
f_{1}:=\frac{\partial f}{\partial \widetilde{L}}, \quad f_{2}:=\frac{\partial f}{\partial \beta}, \quad f_{12}:=\frac{\partial^{2} f}{\partial \widetilde{L} \partial \beta}, \cdots \text { etc. }
$$

where $\widetilde{L}=e^{\sigma} L$. We use the following notations:

$$
\begin{array}{rlrl}
q & :=f f_{2}, & p & :=f f_{1} / L, \\
q_{0} & :=f f_{22}, & p_{0} & :=f_{2}^{2}+q_{0}, \\
q_{-1} & :=f f_{12} / L, \quad p_{-1} & :=q_{-1}+\left(p f_{2} / f\right), \\
q_{-2} & :=f\left(e^{\sigma} f_{11}-\left(f_{1} / L\right)\right) / L^{2}, & p_{-2} & :=q_{-2}+\left(e^{\sigma} p^{2} / f^{2}\right) .
\end{array}
$$

Note that the subscript under each of the above geometric objects indicates the degree of homogeneity of that object. We also use the notations:

$$
m_{i}:=b_{i}-\left(\beta / L^{2}\right) y_{i} \neq 0, \quad p_{02}:=\frac{\partial p_{0}}{\partial \beta} .
$$

Proposition 1.1. Under a generalized $\beta$-conformal change, we have:

(a) $\bar{l}_{i}=e^{\sigma} f_{1} l_{i}+f_{2} b_{i}$,

(b) $\bar{h}_{i j}=e^{\sigma} p h_{i j}+q_{0} m_{i} m_{j}$,

(c) $\bar{g}_{i j}=e^{\sigma} p g_{i j}+p_{0} b_{i} b_{j}+e^{\sigma} p_{-1}\left(b_{i} y_{j}+b_{j} y_{i}\right)+e^{\sigma} p_{-2} y_{i} y_{j}$.

(d) The inverse metric $\bar{g}^{i j}$ of the metric $\bar{g}_{i j}$ is given by

$$
\bar{g}^{i j}=\left(e^{-\sigma} / p\right) g^{i j}-s_{0} b^{i} b^{j}-s_{-1}\left(y^{i} b^{j}+y^{j} b^{i}\right)-s_{-2} y^{i} y^{j},
$$

where

$$
\begin{aligned}
& s_{0}:=e^{-\sigma} f^{2} q_{0} /\left(\varepsilon p L^{2}\right), \quad s_{-1}:=p_{-1} f^{2} /\left(p \varepsilon L^{2}\right), \quad s_{-2}:=p_{-1}\left(e^{\sigma} m^{2} p L^{2}-b^{2} f^{2}\right) /\left(\varepsilon p \beta L^{2}\right), \\
& \varepsilon:=f^{2}\left(e^{\sigma} p+m^{2} q_{0}\right) / L^{2} \neq 0, \quad m^{2}=g^{i j} m_{j} m_{i}=m^{i} m_{i} \neq 0, \quad b^{i}=g^{i j} b_{j} .
\end{aligned}
$$

Remark 1.2. The quantities $s_{0}, s_{-1}, s_{-2}$ satisfy:

$$
\begin{gathered}
\beta s_{0}+L^{2} s_{-1}=q / \varepsilon, \\
b^{2} s_{-1}+\beta s_{-2}=e^{\sigma} p_{-1} m^{2} / \varepsilon .
\end{gathered}
$$

Let $C_{i}=C_{i j k} g^{j k}, C^{i}=C_{j k}^{i} g^{j k}$ and $C^{2}=C^{i} C_{i}$. Then, we have

Proposition 1.3. Under a generalized $\beta$-conformal change, we have

(a) The Cartan tensor $\bar{C}_{i j k}$ has the form

$$
\bar{C}_{i j k}=e^{\sigma} p C_{i j k}+V_{i j k},
$$


(b) The (h)hv-torsion tensor $\bar{C}_{i j}^{l}$ has the form

$$
\bar{C}_{i j}^{l}=C_{i j}^{l}+M_{i j}^{l}
$$

where

$$
\begin{aligned}
V_{i j k}:= & \frac{e^{\sigma} p_{-1}}{2}\left(h_{i j} m_{k}+h_{j k} m_{i}+h_{k i} m_{j}\right)+\frac{p_{02}}{2} m_{i} m_{j} m_{k} \\
M_{i j}^{l}:= & \frac{1}{2}\left(e^{-\sigma} m^{l} / p-m^{2}\left(s_{0} b^{l}+s_{-1} y^{l}\right)\right)\left(p_{02} m_{i} m_{j}+e^{\sigma} p_{-1} h_{i j}\right) \\
& -e^{\sigma}\left(s_{0} b^{l}+s_{-1} y^{l}\right)\left(p C_{i j \beta}+p_{-1} m_{i} m_{j}\right)+\frac{p_{-1}}{2 p}\left(h_{i}^{l} m_{j}+h_{j}^{l} m_{i}\right)
\end{aligned}
$$

and $h_{j}^{i}=g^{i l} h_{l j}, C_{i j \beta}:=C_{i j r} b^{r}, C_{i \beta \beta}:=C_{i j k} b^{j} b^{k}$ and so on.

(c) $\bar{C}_{i}=C_{i}-e^{\sigma} p s_{0} C_{i \beta \beta}+\lambda m_{i}$,

where $\lambda:=\frac{(n+1) p_{-1}}{2 p}-\frac{3 e^{\sigma} p_{-1} m^{2} s_{0}}{2}+\frac{p_{02} m^{2}}{2\left(e^{\sigma} p+q_{0} m^{2}\right)}$,

(d) $\bar{C}^{i}=\frac{e^{-\sigma}}{p} C^{i}+J^{i}$,

where $\quad J^{i}:=\frac{\lambda e^{-\sigma}}{p} m^{i}-s_{0} C_{\beta \beta}^{i}-\left(C_{\beta}+\lambda m^{2}-e^{\sigma} s_{0} p C_{\beta \beta \beta}\right)\left(s_{0} b^{i}+s_{-1} y^{i}\right), C_{\beta}:=C_{i} b^{i}$.

(e) $\bar{C}^{2}=\frac{e^{-\sigma}}{p} C^{2}+\Phi$

where $\Phi:=\lambda^{2} m^{2}\left(\left(e^{-\sigma} / p\right)-s_{0} m^{2}\right)+C_{\beta}\left(\left(2 \lambda e^{-\sigma} / p\right)-s_{0}\left(1+2 \lambda m^{2}\right)\right)$

$$
\begin{aligned}
& +s_{0} C_{\beta \beta \beta}\left(1-3 \lambda+e^{\sigma} s_{0} p C_{\beta}\right) \\
& +s_{0} C_{r \beta \beta}\left(e^{2 \sigma} s_{0}^{2} p^{2} C_{\beta \beta \beta} b^{r}-\lambda s_{0} m^{2} b^{r}-e^{\sigma} s_{0} p C_{\beta \beta}^{r}-2 C^{r}\right) .
\end{aligned}
$$

Proposition 1.4. Under a generalized $\beta$-conformal change, the v-curvature tensor of $(M, L)$ is transformed as follows:

$$
\bar{S}_{l i j k}=e^{\sigma} p S_{l i j k}+\mathfrak{A}_{j k}\left\{H_{l k} h_{i j}+H_{i j} h_{l k}+\omega_{l k} C_{i j \beta}+\omega_{i j} C_{l k \beta}\right\},
$$

where

$$
\begin{aligned}
& H_{i j}:=K_{1} m_{i} m_{k}+K_{2} C_{i j \beta}+K_{3} h_{i j}, \quad \omega_{i j}:=K_{4} m_{i} m_{j}-\frac{1}{2} e^{2 \sigma} p^{2} s_{0} C_{i j \beta}, \\
& K_{1}:=\frac{e^{2 \sigma} p_{-1}^{2}}{4 p}\left(e^{-\sigma}-2 s_{0} p m^{2}\right)+\frac{e^{\sigma} p_{-1} p_{02} m^{2}}{4\left(e^{\sigma} p+q_{0} m^{2}\right)}, \quad K_{2}:=\frac{e^{\sigma} p_{-1}}{2}-\frac{1}{2} e^{2 \sigma} s_{0} p p_{-1} m^{2}, \\
& K_{3}:=\frac{e^{2 \sigma} p_{-1}^{2} m^{2}}{8\left(e^{\sigma} p+q_{0} m^{2}\right)}, \quad K_{4}:=\frac{e^{\sigma} p p_{02}}{2\left(e^{\sigma} p+q_{0} m^{2}\right)}-e^{2 \sigma} s_{0} p p_{-1} .
\end{aligned}
$$

\section{Remark 1.5.}

The tensors $H_{i j}$ and $\omega_{i j}$ defined above have the following properties:

(1) $H_{i j}$ and $\omega_{i j}$ are symmetric.

(2) $H_{i j}$ and $\omega_{i j}$ are indicatory: $H_{i j} y^{i}=0, \omega_{i j} y^{i}=0$. 
(3) $g^{i j} H_{i j}=K_{1} m^{2}+K_{2} C_{\beta}+(n-1) K_{3}$ and $g^{i j} \omega_{i j}=K_{4} m^{2}-\frac{1}{2} e^{2 \sigma} s_{0} p^{2} C_{\beta}$.

Proposition 1.6. Under a generalized $\beta$-conformal change, the vertical Ricci tensor $\bar{S}_{i k}$ and the vertical scalar curvature $\bar{S}$ associated with the transformed space $(M, \bar{L})$ are given by:

$$
\begin{aligned}
\bar{S}_{i k} & =S_{i k}+K h_{i k}+\left(s_{0} m^{2}-\frac{e^{-\sigma}}{(n-3) p}\right) H_{i k}+\Psi_{i k}, \\
\bar{S} & =\frac{e^{-\sigma}}{p} S+\frac{2 e^{-\sigma}}{p} K\left\{(n-2)-e^{\sigma} p s_{0} m^{2}\right\}-s_{0} \Psi_{\beta \beta}+\frac{e^{-\sigma}}{p} \Psi-s_{0} S_{i k} b^{i} b^{k},
\end{aligned}
$$

where

$$
\begin{aligned}
K:= & s_{0} H_{\beta \beta}-\frac{e^{-\sigma}}{p}\left(K_{1} m^{2}+K_{2} C_{\beta}+(n-1) K_{3}\right), \\
\Psi_{i k}:= & \frac{e^{-\sigma}}{p}\left\{\omega_{r k} C_{i \beta}^{r}+\omega_{r i} C_{k \beta}^{r}-\left(K_{4} m^{2}-\frac{1}{2} e^{2 \sigma} s_{0} p^{2} C_{\beta}\right) C_{i k \beta}\right\}-s_{0}\left\{H_{\beta k} m_{i}+H_{i \beta} m_{k}\right. \\
& \left.+\omega_{\beta k} C_{i \beta \beta}+\omega_{i \beta} C_{k \beta \beta}-\omega_{\beta \beta} C_{i k \beta}-\omega_{i k} C_{\beta \beta \beta}+e^{\sigma} p S_{h i j k} b^{h} b^{j}\right\}, \\
H_{\beta \beta}:= & H_{i j} b^{i} b^{j}, \quad \omega_{\beta \beta}:=\omega_{i j} b^{i} b^{j}, \quad \Psi:=\Psi_{i j} g^{i j}, \quad \Psi_{\beta \beta}:=\Psi_{i j} b^{i} b^{j} .
\end{aligned}
$$

Note that the tensor $\Psi_{i j}$ is symmetric and indicatory.

\section{Special Finsler spaces}

In this section we will investigate the effect of the generalized $\beta$-conformal change (1.1) on some special Finsler space. Some of the results obtained in this section are generalizations of known results and some are new. For a systematic study of special Finsler spaces, we refer to [27].

In what follows, let $(M, L)$ be a Finsler manifold and $(M, \bar{L})$ the transformed Finsler manifold under a generalized $\beta$-conformal change. The geometric objects associated with $(M, \bar{L})$ will be denoted by barred symbols.

Theorem 2.1. For $n>2$, under a generalized $\beta$-conformal change, the following assertions are equivalent

(a) $p_{-1}=0$.

(b) $q=k \beta$; $k$ is a nonzero constant.

(c) $\bar{C}_{i j k}=e^{\sigma} p C_{i j k}$.

(d) $\bar{L}=\left(k^{\prime} e^{2 \sigma} L^{2}+k \beta^{2}\right)^{\frac{1}{2}} ; k^{\prime}$ is a nonzero constant.

The special $\beta$-conformal change (d) is referred to as an energy $\beta$-change [28].

Proof.

$(\mathbf{a}) \Rightarrow(\mathbf{b})$ : Let $p_{-1}=0$, then $\frac{f f_{12}}{L}+\frac{p f_{2}}{f}=0$ which leads to $f f_{12}+f_{1} f_{2}=0$, hence, $\frac{\partial}{\partial \widetilde{L}}\left(f f_{2}\right)=0$. By integration, taking the homogeneity of $f$ into account, we get 
$q=k \beta$, with $k \neq 0$.

(b) $\Rightarrow(\mathbf{c})$ : Let $q=k \beta$, then $\frac{\partial}{\partial \widetilde{L}}\left(f f_{2}\right)=f f_{12}+f_{1} f_{2}=0$, which leads to $p_{-1}=0$. Using $\beta p_{0}+e^{\sigma} L^{2} p_{-1}=q$, we get $\beta p_{0}=q$. By differentiating the last identity with respect to $\beta$, we have

$$
\beta p_{02}+p_{0}=f_{2}^{2}+f f_{22}=p_{0},
$$

which leads to $p_{02}=0$. Hence, by (1.4) $V_{i j k}=0$ and, consequently, $\bar{C}_{i j k}=e^{\sigma} p C_{i j k}$.

$(\mathbf{c}) \Rightarrow(\mathbf{d})$ : Let $V_{i j k}=0$, then

$$
e^{\sigma} p_{-1}\left(h_{i j} m_{k}+h_{j k} m_{i}+h_{k i} m_{j}\right)+p_{02} m_{i} m_{j} m_{k}=0 .
$$

By contraction by $b^{i}$, we have

$$
e^{\sigma} p_{-1}\left(2 m_{j} m_{k}+m^{2} h_{j k}\right)+p_{02} m^{2} m_{j} m_{k}=0 .
$$

Contracting (2.1) again by $b^{j}$, we get $3 e^{\sigma} p_{-1}=m^{2} p_{02}$. Hence, (2.1) reduces to $p_{-1}\left(m^{2} h_{j k}-m_{j} m_{k}\right)=0$, which leads to $p_{-1}=0$ or $m^{2} h_{j k}-m_{j} m_{k}=0$. Now, if $m^{2} h_{j k}-m_{j} m_{k}=0$, then, $n=2$ which contradicts the hypothesis. Hence, $p_{-1}=0$, and consequently, $q=k \beta$. Then, we have the partial differential equation

$$
f f_{2}=k \beta
$$

By integration with respect to $\beta$ and using the fact that $f$ is homogenous of degree 1 in $\beta$ and $\widetilde{L}$, we get

$$
f^{2}=k \beta^{2}+\varphi(\widetilde{L})
$$

where $\varphi(\widetilde{L})$ is a homogenous function of degree 2 in $\widetilde{L}$, which may be written as $\varphi(\widetilde{L})=k^{\prime} \widetilde{L}^{2}$. Hence, $f^{2}=k \beta^{2}+k^{\prime} \widetilde{L}^{2}$ and consequently,

$$
\bar{L}=\left(k^{\prime} \widetilde{L}^{2}+k \beta^{2}\right)^{\frac{1}{2}}=\left(k^{\prime} e^{2 \sigma} L^{2}+k \beta^{2}\right)^{\frac{1}{2}} .
$$

$(\mathrm{d}) \Rightarrow(\mathrm{a})$ : It is obvious.

Corollary 2.2. For $n>2$, under a generalized $\beta$-conformal change, if one of the above equivalent conditions holds, then the space $(M, \bar{L})$ is Riemannian if and only if $(M, L)$ is Riemannian.

We will study the change of some special Finsler spaces under a generalized $\beta$ conformal change.

Definition 2.3. A Finsler space $(M, L)$ with dimension $n \geq 3$ is said to be quasi-Creducible if the Cartan tensor $C_{i j k}$ satisfies

$$
C_{i j k}=Q_{i j} C_{k}+Q_{j k} C_{i}+Q_{k i} C_{j}
$$

where $Q_{i j}$ is a symmetric indicatory tensor. 
By Proposition 1.3, assuming $\lambda \neq 0$, we have

$$
\begin{aligned}
\bar{C}_{i j k}= & e^{\sigma} p C_{i j k}+\frac{e^{\sigma} p_{-1}}{2}\left(h_{i j} m_{k}+h_{j k} m_{i}+h_{k i} m_{j}\right)+\frac{p_{02}}{2} m_{i} m_{j} m_{k} \\
= & e^{\sigma} p C_{i j k}+\frac{1}{6} \mathfrak{S}_{i j k}\left\{\left(3 e^{\sigma} p_{-1} h_{i j}+p_{02} m_{i} m_{j}\right) m_{k}\right\} \\
= & e^{\sigma} p C_{i j k}+\frac{1}{6 \lambda} \mathfrak{S}_{i j k}\left\{\left(3 e^{\sigma} p_{-1} h_{i j}+p_{02} m_{i} m_{j}\right)\left(\bar{C}_{k}-C_{k}+e^{\sigma} p s_{0} C_{k \beta \beta}\right)\right\} \\
= & e^{\sigma} p C_{i j k}+\frac{1}{6 \lambda} \mathfrak{S}_{i j k}\left\{\left(3 e^{\sigma} p_{-1} h_{i j}+p_{02} m_{i} m_{j}\right) \bar{C}_{k}\right\} \\
& +\frac{1}{6 \lambda} \mathfrak{S}_{i j k}\left\{\left(3 e^{\sigma} p_{-1} h_{i j}+p_{02} m_{i} m_{j}\right)\left(e^{\sigma} p s_{0} C_{k \beta \beta}-C_{k}\right)\right\} .
\end{aligned}
$$

Hence, we have

Lemma 2.4. Under a generalized $\beta$-conformal change, the transformed Cartan tensor can be written in the form

$$
\bar{C}_{i j k}=\mathfrak{S}_{i j k}\left\{\bar{Q}_{i j} \bar{C}_{k}\right\}+q_{i j k},
$$

where $\bar{Q}_{i j}:=\frac{1}{6 \lambda}\left(3 e^{\sigma} p_{-1} h_{i j}+p_{02} m_{i} m_{j}\right)$,

$$
q_{i j k}:=\frac{1}{6 \lambda} \mathfrak{S}_{i j k}\left\{2 e^{\sigma} \lambda p C_{i j k}+\left(3 e^{\sigma} p_{-1} h_{i j}+p_{02} m_{i} m_{j}\right)\left(e^{\sigma} p s_{0} C_{k \beta \beta}-C_{k}\right)\right\} .
$$

By the above lemma and taking into account that the tensor $\bar{Q}_{i j}$ is symmetric and indicatory, we get the following result.

Theorem 2.5. If the tensor $q_{i j k}=0$, then the space $(M, \bar{L})$ is quasi-C-reducible.

As a corollary of the above theorem, we obtain a generalized form of Matsumoto's result [14]:

Corollary 2.6. Under a generalized $\beta$-conformal change, a Reimannian space $(M, L)$ is transformed to a quasi-C-reducible space.

Definition 2.7. A Finsler space $(M, L)$ of dimension $n \geq 3$ is called semi-C-reducible, if the Cartan tensor $C_{i j k}$ is written in the form:

$$
C_{i j k}=\frac{r}{n+1}\left(h_{i j} C_{k}+h_{k i} C_{j}+h_{j k} C_{i}\right)+\frac{t}{C^{2}} C_{i} C_{j} C_{k}
$$

where $r$ and $t$ are scalar functions such that $r+t=1$.

The next result has been obtained by Matsumoto and Shibata [16] in the special case of Finsler spaces with $(\alpha, \beta)$-metric.

Theorem 2.8. A Riemannian space is transformed to a semi-C-reducible space, by a generalized $\beta$-conformal change. 
Proof. From Proposition 1.1 and Proposition 1.3, we get

$$
\begin{aligned}
\bar{C}_{i j k} & =\frac{1}{2} e^{\sigma} p_{-1}\left(h_{i j} m_{k}+h_{j k} m_{i}+h_{k i} m_{j}\right)+\frac{1}{2} p_{02} m_{i} m_{j} m_{k} \\
& =\frac{p_{-1}}{2 p \lambda}\left(\bar{h}_{i j} \bar{C}_{k}+\bar{h}_{j k} \bar{C}_{i}+\bar{h}_{k i} \bar{C}_{k} j\right)+\frac{m^{2}\left(p p_{02}-3 p_{-1} q_{0}\right)}{2 p \lambda\left(e^{\sigma} p+m^{2} q_{0}\right) \bar{C}^{2}} \bar{C}_{i} \bar{C}_{j} \bar{C}_{k} \\
& =\frac{r}{n+1}\left(\bar{h}_{i j} \bar{C}_{k}+\bar{h}_{j k} \bar{C}_{i}+\bar{h}_{k i} \bar{C}_{k} j\right)+\frac{t}{\bar{C}^{2}} \bar{C}_{i} \bar{C}_{j} \bar{C}_{k},
\end{aligned}
$$

where

$$
r=\frac{p_{-1}(n+1)}{2 p \lambda}, \quad t=\frac{m^{2}\left(p p_{02}-3 p_{-1} q_{0}\right)}{2 p \lambda\left(e^{\sigma} p+m^{2} q_{0}\right)}, \quad r+t=1,
$$

which means that $(M, \bar{L})$ is semi-reducible.

Definition 2.9. A Finsler space $(M, L)$ of dimension $n \geq 3$ is called C-reducible if the Cartan tensor $C_{i j k}$ has the form:

$$
C_{i j k}=h_{i j} A_{k}+h_{k i} A_{j}+h_{j k} A_{i}, \quad A_{i}=\frac{C_{i}}{n+1} .
$$

Define the tensor

$$
K_{i j k}=C_{i j k}-\left(h_{i j} A_{k}+h_{k i} A_{j}+h_{j k} A_{i}\right) .
$$

It is clear that $K_{i j k}$ is symmetric and indicatory. Moreover, $K_{i j k}$ vanishes if and only if the Finsler space $(M, L)$ is C-reducible.

Proposition 2.10. Under a generalized $\beta$-conformal change, the tensor $\bar{K}_{i j k}$ associated with the space $(M, \bar{L})$ has the form

$$
\bar{K}_{i j k}=e^{\sigma} p K_{i j k}+d_{i j k}
$$

where

$$
\begin{aligned}
d_{i j k}:= & \frac{1}{n+1} \mathfrak{S}_{i j k}\left\{(n+1)\left(\alpha_{1} h_{i j}+\alpha_{2} m_{i} m_{j}\right) m_{k}+q_{0} m_{i} m_{j} C_{k}\right. \\
& \left.+\left(s_{0} p q_{0} m_{i} m_{j}+e^{\sigma} p^{2} s_{0} h_{i j}\right) C_{k \beta \beta}\right\}, \\
\alpha_{1}:= & \frac{e^{\sigma} p_{-1}}{2}-\frac{e^{\sigma} p \lambda}{n+1}, \quad \alpha_{2}:=\frac{p_{02}}{6}-\frac{q_{0} \lambda}{n+1} .
\end{aligned}
$$

Consequently, we have

Theorem 2.11. Under a generalized $\beta$-conformal change, the following assertions

(a) the space $(M, L)$ is C-reducible,

(b) the space $(M, \bar{L})$ is C-reducible

are equivalent if and only if the tensor $d_{i j k}$ vanishes.

Corollary 2.12. If $\bar{L}=e^{\sigma} L+\beta, L$ being Finslerian, then the tensor $d_{i j k}$ vanishes. Consequently, $(M, \bar{L})$ is C-reducible if and only if $(M, L)$ is C-reducible. 
Lemma 2.13. Under a generalized $\beta$-conformal change $\bar{L}=f\left(e^{\sigma} L, \beta\right)$, with $L$ Riemannian, the tensor $d_{i j k}$ takes the form

$$
d_{i j k}=\mathfrak{S}_{i j k}\left\{\alpha_{1} h_{i j} m_{k}+\alpha_{2} m_{i} m_{j} m_{k}\right\} .
$$

Theorem 2.14. Under a generalized $\beta$-conformal change $\bar{L}=f\left(e^{\sigma} L, \beta\right)$, with $L$ Riemannian, the following assertions are equivalent:

(a) $\alpha_{1}=0$ and $\alpha_{2}=0$,

(b) $(M, \bar{L})$ is C-reducible,

(c) $(M, \bar{L})$ is either of Randers type or of Kropina type.

Proof.

(a) $\Rightarrow($ b): It is obvious.

(b) $\Rightarrow(\mathbf{a})$ : Let the space $(M, \bar{L})$ be C-reducible, then, by Lemma 2.13, we have:

$$
d_{i j k}=\mathfrak{S}_{i j k}\left\{\alpha_{1} h_{i j} m_{k}+\alpha_{2} m_{i} m_{j} m_{k}\right\}=0 .
$$

Contracting (2.5) by $g^{i j}$, we get

$$
(n+1) \alpha_{1}+3 m^{2} \alpha_{2}=0
$$

and contracting the same equation by $b^{i} b^{j}$, we get

$$
\alpha_{1}+m^{2} \alpha_{2}=0
$$

The last two relations lead to $(n-2) \alpha_{1}=0$. Since $n>2$, then $\alpha_{1}=0$ and consequently $\alpha_{2}=0$, by (2.7).

(a) $\Rightarrow(\mathbf{c})$ : If $\alpha_{1}=\alpha_{2}=0$, we have $(n+1) p_{-1}=2 p \lambda$ and $(n+1) p_{02}=6 q_{0} \lambda$. Solving the last two equations for $\lambda$, we get

$$
3 q_{0} p_{-1}=p p_{02}
$$

From which we obtain the patrial differential equation

$$
\frac{3 f_{12} f_{22}}{f_{1}}-f_{222}=0
$$

Now, if $f_{22}=0$, by integration with respect to $\beta$ and taking the homogeneity of $f$ into account, we get $f_{2}=\varphi_{1}(\widetilde{L})$, where $\varphi_{1}(\widetilde{L})$ is a homogenous function of degree 0 in $\widetilde{L}$. Hence, by integrating $f_{2}$ with respect to $\beta$, we get

$$
\bar{L}=\varphi_{1}(\widetilde{L}) \beta+\varphi_{2}(\widetilde{L})
$$

where $\varphi_{2}(\widetilde{L})$ is a homogenous function of degree 1 in $\widetilde{L}$. By the homogeneity properties of $\varphi_{1}(\widetilde{L})$ and $\varphi_{2}(\widetilde{L})$, using Euler theorem, we conclude that $\varphi_{1}(\widetilde{L})=c_{1}$ and $\varphi_{2}(\widetilde{L})=$ $c_{2}$, where $c_{1}$ and $c_{2}$ are constants. Consequently,

$$
\bar{L}=c_{2} \widetilde{L}+c_{1} \beta
$$


On the other hand, if $f_{22} \neq 0$, we have

$$
\frac{3 f_{12}}{f_{1}}-\frac{f_{222}}{f_{22}}=0
$$

which, by integration with respect to $\beta$, gives

$$
3 \ln f_{1}-\ln f_{22}=\ln \varphi_{3}(\widetilde{L}) \Rightarrow \frac{f_{1}^{3}}{f_{22}}=\varphi_{3}(\widetilde{L})=c_{3} \widetilde{L}
$$

where $\varphi_{3}(\widetilde{L})$ is a homogenous function of degree 1 in $\widetilde{L}$ and $c_{3}$ is nonzero constant. Using $\widetilde{L} f_{11}+\beta f_{12}=0$ and $\widetilde{L} f_{21}+\beta f_{22}=0$, we have

$$
\frac{f_{11}}{f_{1}^{3}}=\frac{c_{3} \beta^{2}}{\widetilde{L}^{3}}
$$

from which $f_{1}=\frac{\widetilde{L}}{\sqrt{c_{3} \beta^{2}+c_{4} \widetilde{L}^{2}}}$. If $c_{4} \neq 0$, then

$$
f=\frac{1}{c_{4}} \sqrt{c_{3} \beta^{2}+c_{4} \widetilde{L}^{2}}+c_{5} \beta
$$

and if $c_{4}=0$, then

$$
f=\frac{\widetilde{L}^{2}+\beta^{2}}{c_{3} \beta} .
$$

The former may be regarded as of Randers type and the later as of Kropina type.

(c) $\Rightarrow(\mathbf{a})$ : The result follows directly by computing $\alpha_{1}$ and $\alpha_{2}$ for Randers and Kropina spaces.

It should be noted that Matsumoto [9] showed that C-reducible Finsler spaces with $(\alpha, \beta)$-metric are either of Randers type or of Kropina type.

Definition 2.15. A Finsler space $(M, L)$ of dimension $n \geq 2$ is said to be $C_{2}$-like if the Cartan tensor $C_{i j k}$ satisfies

$$
C^{2} C_{i j k}=C_{i} C_{j} C_{k}
$$

Let us define the tensor

$$
\eta_{i j k}=C^{2} C_{i j k}-C_{i} C_{j} C_{k}
$$

It is clear that $\eta_{i j k}$ is symmetric and indicatory. Moreover, $\eta_{i j k}$ vanishes if and only if the Finsler space is $C_{2}$-like space.

Proposition 2.16. Under a generalized $\beta$-conformal change, the tensor $\bar{\eta}_{i j k}$ associated with the space $(M, \bar{L})$ has the form

$$
\bar{\eta}_{i j k}=\eta_{i j k}+I_{i j k}
$$


where

$$
\begin{aligned}
I_{i j k}:= & \left(e^{-\sigma} / p\right) C^{2} V_{i j k}+\Phi\left(e^{\sigma} p C_{i j k}+V_{i j k}\right)-\lambda^{3} m_{i} m_{j} m_{k} \\
& -\lambda^{2}\left(m_{j} m_{k} C_{i}+m_{i} m_{j} C_{k}+m_{i} m_{k} C_{j}\right)-\lambda\left(m_{k} C_{i} C_{j}+m_{j} C_{k} C_{i}+m_{i} C_{j} C_{k}\right) \\
& -e^{\sigma} p s_{0}\left[C _ { i \beta \beta } \left\{e^{\sigma} p s_{0} C_{k \beta \beta}\left(\lambda m_{j}-C_{j}-e^{\sigma} p s_{0} C_{j \beta \beta}\right)+e^{\sigma} p s_{0} C_{j \beta \beta}\left(\lambda m_{k}+C_{k}\right)\right.\right. \\
& \left.-\lambda\left(m_{k} C_{j}+m_{j} C_{k}-\lambda m_{j} m_{k}\right)-C_{j} C_{k}\right\}+C_{k \beta \beta}\left(C_{i} C_{j}-e^{\sigma} p s_{0} C_{i} C_{j \beta \beta}+\lambda m_{i} C_{j}\right. \\
& \left.\left.+\lambda m_{j} C_{i}+\lambda^{2} m_{i} m_{j}\right)+\lambda C_{j \beta \beta}\left(\lambda m_{i} m_{k}+C_{i} C_{k}+m_{i} C_{k}+m_{k} C_{i}-e^{\sigma} p s_{0} m_{i} C_{k \beta \beta}\right)\right] .
\end{aligned}
$$

Theorem 2.17. Under a generalized $\beta$-conformal change, the following assertions

(a) the space $(M, L)$ is $C_{2}$-like,

(b) the space $(M, \bar{L})$ is $C_{2}$-like

are equivalent if and only if the tensor $I_{i j k}$ vanishes.

Lemma 2.18. Starting with a Riemannian space $(M, L)$, under a generalized $\beta$ conformal change, the tensor $I_{i j k}$ takes the form:

$$
I_{i j k}=\Phi V_{i j k}-\lambda^{3} m_{i} m_{j} m_{k} .
$$

Theorem 2.19. For a $\beta$-conformal change $\bar{L}=e^{\sigma} L+\beta ; L$ being Finslerian, $a$ necessary condition for the assertions

(a) the space $(M, L)$ is $C_{2}$-like,

(b) the space $(M, \bar{L})$ is $C_{2}$-like

to be equivalent is that $C_{\beta}=0$.

Proof. In the case of $\bar{L}=e^{\sigma} L+\beta$; L being Finslerian, $\Phi=\lambda e^{-\sigma} L\left(\lambda m^{2}+2 C_{\beta}\right) / \bar{L}$, $\lambda=\frac{n+1}{2 \bar{L}}$ and $V_{i j k}=\frac{e^{\sigma}}{2 L}\left(h_{i j} m_{k}+h_{j k} m_{i}+h_{k i} m_{j}\right)$. Now, let the above assertions be equivalent, so $I_{i j k}=0$. Contracting (2.9) by $g^{j k}$, we have $\frac{n+1}{2 \bar{L}} C_{\beta}=0$ and the result follows.

If $(M, L)$ is a Riemannian space and the tensor $I_{i j k}$ vanishes, i.e., $(M, \bar{L})$ is $C_{2}$-like, we have

$$
\lambda^{2} \frac{m^{2}}{e^{\sigma} p+q_{0} m^{2}}\left(e^{\sigma} p_{-1}\left(h_{i j} m_{k}+h_{j k} m_{i}+h_{k i} m_{j}\right)+p_{02} m_{i} m_{j} m_{k}\right)-2 \lambda^{3} m_{i} m_{j} m_{k}=0,
$$

contracting by $b^{i} b^{j}$ and assuming that $\lambda \neq 0$, we get

$$
(n-2) p_{-1}=0 \text {. }
$$

Hence, we have

Theorem 2.20. Starting with a Riemannian space $(M, L)$, if the transformed space $(M, \bar{L})$ is $C_{2}$-like, then one of the following holds:

(a) $\operatorname{dim} M=2$. 
(b) The generalized $\beta$-conformal change is an energy $\beta$-change and the transformed space is Rimannian.

Corollary 2.21. Let the generalized $\beta$-conformal change be of the form $\bar{L}=e^{\sigma} L+\beta$, with L Riemannian. If $(M, \bar{L})$ is $C_{2}$-like, then $\operatorname{dim} M=2$.

Corollary 2.22. A Reimannian space of dimension $\geq 3$ can not be transformed to a non-Reimannian $C_{2}$-like space.

Now, we are going to study two special Finsler spaces whose defining property depends on the v-curvature tensor $S_{l i j k}$, namely, the $S_{3}$-like and $S_{4}$-like Finsler spaces.

Definition 2.23. A Finsler space $\left(M^{n}, L\right)$ with dimension $n>3$ is said to be $S_{3}$-like if the v-curvature tensor $S_{l i j k}$ satisfies

$$
S_{l i j k}=\frac{S}{(n-1)(n-2)}\left\{h_{i k} h_{l j}-h_{i j} h_{l k}\right\},
$$

where $S$ is the vertical scalar curvature.

Define the following tensor

$$
\mu_{l i j k}=S_{l i j k}-\frac{S}{(n-1)(n-2)}\left\{h_{i k} h_{l j}-h_{i j} h_{l k}\right\} .
$$

It is clear that the tensor $\mu_{h i j k}$ vanishes if and only if the space is $S_{3}$-like.

Proposition 2.24. Under a generalized $\beta$-conformal change, the tensor $\bar{\mu}_{\text {hijk }}$ associated with the space $(M, \bar{L})$ has the form:

$$
\bar{\mu}_{l i j k}=e^{\sigma} p \mu_{l i j k}+r_{l i j k}
$$

where

$$
\begin{aligned}
r_{l i j k}= & \mathfrak{A}_{j k}\left\{H_{l k} h_{i j}+H_{i j} h_{l k}+\omega_{l k} C_{\beta i j}+\omega_{i j} C_{\beta l k}-\frac{e^{2 \sigma} p^{2} \Omega}{(n-1)(n-2)} h_{l j} h_{i k}\right. \\
& \left.-\frac{q_{0}}{(n-1)(n-2)}\left(S+e^{\sigma} p \Omega\right)\left(h_{i k} m_{l} m_{j}+h_{l j} m_{i} m_{k}\right)\right\}, \\
\Omega:= & \frac{e^{-\sigma}}{p} \Psi-s_{0} S_{i k} b^{i} b^{k}-s_{0} \Psi_{\beta \beta}+\frac{2 e^{-\sigma}}{p} K\left(n-2-e^{\sigma} p s_{0} m^{2}\right) .
\end{aligned}
$$

Theorem 2.25. Under a generalized $\beta$-conformal change, the following assertions

(a) the space $(M, L)$ is $S_{3}$-like,

(b) the space $(M, \bar{L})$ is $S_{3}$-like

are equivalent if and only if the tensor $r_{l i j k}$ vanishes.

Proposition 2.26. For a $\beta$-conformal change $\bar{L}=e^{\sigma} L+\beta$, the tensor $r_{\text {lijk }}$ takes the form

$$
r_{l i j k}=C_{\beta j k} h_{i l}+\frac{1}{2 \bar{L}} m_{j} m_{k} h_{i l}+\frac{m^{2}}{4 \bar{L}} h_{j k} h_{i l}-A_{\beta} h_{j k} h_{i l},
$$

where $A_{\beta}=\frac{1}{n-1}\left(C_{\beta}+\frac{n+1}{4 \bar{L}} m^{2}\right)$. 
From the above proposition, we retrieve a result due to Abed [1]:

Corollary 2.27. In the case of a $\beta$-conformal change $\bar{L}=e^{\sigma} L+\beta$, the following assertions

(a) the space $(M, L)$ is $S_{3}$-like,

(b) the space $(M, \bar{L})$ is $S_{3}$-like

are equivalent if and only if

$$
C_{r j k} b^{r}+\frac{1}{2 \bar{L}} m_{j} m_{k}+\frac{m^{2}}{4 \bar{L}} h_{j k}=A_{\beta} h_{j k} .
$$

Finally, we study $S_{4}$-like Finsler spaces under a generalized $\beta$-conformal change.

Definition 2.28. A Finsler space $(M, L)$ with dimension $n>4$ is said to be $S_{4}$-like if the v-curvature tensor $S_{\text {hijk }}$ satisfies

$$
S_{l i j k}=\mathfrak{A}_{j k}\left\{h_{l j} \mathbb{M}_{i k}+h_{i k} \mathbb{M}_{l j}\right\}
$$

where $\mathbb{M}_{i k}=\frac{1}{(n-3)}\left\{S_{i k}-\frac{S h_{i k}}{2(n-2)}\right\}$.

Define the tensor

$$
\zeta_{l i j k}=S_{l i j k}-\mathfrak{A}_{j k}\left\{h_{l j} \mathbb{M}_{i k}+h_{i k} \mathbb{M}_{l j}\right\}
$$

It is clear that the tensor $\zeta_{h i j k}$ vanishes if and only if the space is $S_{4}$-like.

Proposition 2.29. Under a generalized generalized $\beta$-conformal change, the tensor $\bar{\zeta}_{\text {hijk }}$ associated with the space $(M, \bar{L})$ has the form

$$
\bar{\zeta}_{l i j k}=e^{\sigma} p \zeta_{l i j k}+\varepsilon_{l i j k}
$$

where

$$
\begin{aligned}
\varepsilon_{l i j k}:= & \mathfrak{F}_{l i j k}\left\{\omega_{l k} C_{i j s} b^{s}+q_{0} \mathbb{M}_{l k} m_{i} m_{j}+\frac{e^{\sigma} p}{n-3}\left(s_{0} m^{2} H_{l k} h_{i j}+K h_{l k} h_{i j}+\Psi_{l k} h_{i j}\right)\right. \\
& -\frac{e^{\sigma}}{n-3}\left(\frac{e^{-\sigma} q_{0} S}{2(n-2)} m_{l} m_{k} h_{i j}+\frac{\Omega p\left(e^{\sigma} p h_{l k}+q_{0} m_{l} m_{k}\right)}{2(n-2)} h_{i j}+e^{-\sigma} q_{0} K h_{l k} m_{i} m_{j}\right) \\
& \left.+\frac{q_{0}}{(n-3) p}\left(\Psi_{l k}+\left(s_{0} p m^{2}-(n-3) e^{-\sigma}\right) H_{l k}-\frac{\Omega e^{\sigma} p^{2}}{2(n-2)} h_{l k}\right) m_{i} m_{j}\right\},
\end{aligned}
$$

and

$$
\mathfrak{F}_{l i j k}\left\{X_{l k} Y_{i j}\right\}:=X_{l k} Y_{i j}+X_{i j} Y_{l k}-X_{l j} Y_{i k}-X_{i k} Y_{l j}
$$

Theorem 2.30. Under a generalized $\beta$-conformal change, the following assertions

(a) the space $(M, L)$ is $S_{4}$-like,

(b) the space $(M, \bar{L})$ is $S_{4}$-like 
are equivalent if and only if the tensor $\varepsilon_{h i j k}$ vanishes.

In the case of a $\beta$-conformal change $\bar{L}=e^{\sigma} L+\beta$, the tensor $\varepsilon_{h i j k}$ vanishes and we retrieve the the following result of Abed [1].

Corollary 2.31. For a of $\beta$-conformal change $\bar{L}=e^{\sigma} L+\beta$, the space $(M, L)$ is $S_{4}$-like if and only if the space $(M, \bar{L})$ is $S_{4}$-like.

\section{The T-tensor $T_{h i j k}$}

The T-tensor is defined by 10

$$
T_{h i j k}=\left.L C_{h i j}\right|_{k}+C_{h i j} l_{k}+C_{h i k} l_{j}+C_{h j k} l_{i}+C_{i j k} l_{h},
$$

It should be noted that the T-tensor has a great contribution in geometric properties of special Finsler spaces. For instance, Hashiguchi [4] has shown that a Landsberg space remains Landsberg under a conformal transformation, if and only if $T_{h i j k}=0$. On the other hand, Matsumoto [12 has obtained interesting results for spaces with $T_{h i j k}=0$ and, further, he investigated the three-dimensional Finsler spaces with vanishing $\mathrm{T}$-tensor.

In this section we compute the T-tensor under a generalized $\beta$-conformal change and consider some interesting special cases.

Theorem 3.1. Under a generalized $\beta$-conformal change, the transformed T-tensor takes the form:

$$
\begin{aligned}
\bar{T}_{l i j k}= & \frac{e^{\sigma} p \bar{L}}{L} T_{l i j k}-\bar{L}\left(\frac{\beta e^{\sigma} p_{-1}}{2 L^{2}}+2 K_{3}\right)\left(h_{l i} h_{j k}+h_{l j} h_{i k}+h_{l k} h_{i j}\right) \\
& +\left(h_{l i} \nu_{j k}+h_{l j} \nu_{i k}+h_{i j} \nu_{l k}+h_{j k} \nu_{i l}+h_{l k} \nu_{i j}+h_{i k} \nu_{j l}\right) \\
& +\left(e^{\sigma} p f_{2}-\frac{1}{2} e^{\sigma} \bar{L}_{-1}\right)\left(C_{l i j} m_{k}+C_{i j k} m_{l}+C_{j l k} m_{i}+C_{l i k} m_{j}\right) \\
& -\bar{L}\left(M_{i j} C_{l k \beta}+M_{j l} C_{i k \beta}+M_{i l} C_{j k \beta}+M_{l k} C_{i j \beta}+M_{j k} C_{i l \beta}+M_{i k} C_{j l \beta}\right) \\
& +\bar{L} e^{2 \sigma} s_{0} p^{2}\left(C_{i j \beta} C_{l k \beta}+C_{l j \beta} C_{i k \beta}+C_{i l \beta} C_{j k \beta}\right)+\frac{1}{2} \bar{L}\left(6 K_{5}+p_{022}\right) m_{l} m_{i} m_{j} m_{k} \\
& \bar{L} p_{02}\left(n_{i j} m_{k} m_{l}+n_{l k} m_{j} m_{j}\right)+\frac{1}{2} p_{02}\left(\dot{n}_{i j} m_{k} m_{l}+\dot{n}_{l k} m_{i} m_{j}\right)
\end{aligned}
$$

where

$$
\begin{aligned}
\nu_{i j} & :=\frac{1}{2} e^{\sigma} p_{-1} \dot{n}_{i j}-\bar{L}\left(K_{1}+\frac{3 e^{\sigma} p_{-1}^{2}}{4 p}+\frac{\beta p_{02}}{2 L^{2}}\right) m_{i} m_{j}-\frac{\bar{L} e^{\sigma} p_{-1}}{2 L} n_{i j}, \\
K_{5} & :=e^{2 \sigma} s_{0} p_{-1}^{2}-\frac{4 e^{\sigma} p_{-1} p_{02}+p_{02}^{2} m^{2}}{4\left(e^{\sigma} p+q_{0} m^{2}\right)} \\
\dot{n}_{i j} & :=\bar{l}_{i} m_{j}+\bar{l}_{j} m_{i} .
\end{aligned}
$$


Proof. One can show that

$$
\begin{aligned}
\dot{\partial}_{k} \bar{C}_{l i j}= & e^{\sigma} p \dot{\partial}_{k} C_{l i j}+e^{\sigma} p_{-1}\left(C_{l i j} m_{k}+C_{i j k} m_{l}+C_{j l k} m_{i}+C_{l i k} m_{j}\right) \\
& -\frac{e^{\sigma} p_{-1}}{2 L}\left(h_{l i} n_{j k}+h_{l j} n_{i k}+h_{i j} n_{l k}+h_{j k} n_{l i}+h_{l k} n_{i j}+h_{i k} n_{j l}\right) \\
& -\frac{\beta e^{\sigma} p_{-1}}{2 L^{2}}\left(h_{l i} h_{j k}+h_{l j} h_{i k}+h_{l k} h_{i j}\right)-\frac{\beta p_{02}}{2 L^{2}}\left(h_{i j} m_{k} m_{l}+h_{l i} m_{j} m_{k}+h_{k l} m_{i} m_{j}\right. \\
& \left.+h_{j k} m_{l} m_{i}+h_{i k} m_{j} m_{l}+h_{l j} m_{i} m_{k}\right)+\frac{1}{2} p_{022} m_{i} m_{j} m_{k} m_{l} \\
& -\frac{p_{02}}{2 L}\left(n_{i j} m_{k} m_{l}+n_{l k} m_{i} m_{j}\right)
\end{aligned}
$$

where $n_{i j}:=l_{i} m_{j}+l_{j} m_{i}$ and $p_{022}:=\frac{\partial}{\partial \beta} p_{02}$. Similarly,

$$
\begin{aligned}
\bar{C}_{i j r} \bar{C}_{l k}^{r}= & e^{\sigma} p C_{i j r} C_{l k}^{r}+\frac{1}{2} e^{\sigma} p_{-1}\left(C_{l j k} m_{i}+C_{i l k} m_{j}+C_{i j k} m_{l}+C_{i j l} m_{k}\right) \\
& +K_{4}\left(C_{l k \beta} m_{i} m_{j}+C_{i j \beta} m_{l} m_{k}\right)+\left(K_{1}+\frac{1}{4 p} e^{\sigma} p_{-1}^{2}\right)\left(h_{i j} m_{k} m_{l}+h_{l k} m_{i} m_{j}\right) \\
& +2 K_{3} h_{i j} h_{l k}+K_{2}\left(C_{l k \beta} h_{i j}+C_{i j \beta} h_{l k}\right)-K_{5} m_{i} m_{j} m_{l} m_{k}-e^{2 \sigma} p^{2} s_{0} C_{i j \beta} C_{l k \beta} \\
& +\frac{e^{\sigma} p_{-1}^{2}}{4 p}\left(h_{l i} m_{j} m_{k}+h_{j k} m_{h} m_{i}+h_{i k} m_{j} m_{h}+h_{j l} m_{i} m_{k}\right)
\end{aligned}
$$

Using (3.1) and (3.2), we get

$$
\begin{aligned}
\bar{C}_{l i j} \overline{\mid}_{k}= & \dot{\partial}_{k} \bar{C}_{l i j}-\bar{C}_{l k}^{m} \bar{C}_{m i j}-\bar{C}_{i k}^{m} \bar{C}_{m l j}-\bar{C}_{j k}^{m} \bar{C}_{m l i} \\
= & \left.e^{\sigma} p C_{l i j}\right|_{k}-\left(\frac{\beta e^{\sigma} p_{-1}}{2 L^{2}}+2 K_{3}\right)\left(h_{l i} h_{j k}+h_{l j} h_{i k}+h_{l k} h_{i j}\right)+3 K_{5} m_{i} m_{j} m_{k} m_{l} \\
& +\frac{1}{2} e^{\sigma} p_{-1}\left(C_{l j k} m_{i}+C_{i l k} m_{j}+C_{i j k} m_{l}+C_{i j l} m_{k}\right)-\frac{p_{02}}{2 L}\left(n_{i j} m_{k} m_{l}+n_{l k} m_{j} m_{j}\right) \\
& -\frac{e^{\sigma} p_{-1}}{2 L}\left(h_{l i} n_{j k}+h_{l j} n_{i k}+h_{i j} n_{l k}+h_{j k} n_{l k}+h_{i j} n_{l k}+h_{i k} n_{j l}\right) \\
& -\left(K_{1}+\frac{3 e^{\sigma} p_{-1}^{2}}{4 p}+\frac{\beta p_{02}}{2 L^{2}}\right)\left(h_{i j} m_{l} m_{k}+h_{l k} m_{i} m_{j}+h_{l i} m_{j} m_{k}+h_{j k} m_{l} m_{i}\right. \\
& \left.+h_{i k} m_{j} m_{l}+h_{j l} m_{i} m_{k}\right)-\left(M_{i j} C_{l k \beta}+M_{j l} C_{l i k \beta}+M_{i l} C_{j k \beta}+M_{l k} C_{i j \beta}\right. \\
& \left.+M_{j k} C_{i l \beta}+M_{i k} C_{j l \beta}\right)+e^{2 \sigma} s_{0} p^{2}\left(C_{i j \beta} C_{l k \beta}+C_{l j \beta} C_{i k \beta}+C_{i l \beta} C_{j k \beta}\right),
\end{aligned}
$$

where $M_{i j}:=K_{2} h_{i j}+K_{4} m_{i} m_{j}$.

The result follows from (3.3), Proposition 1.3 and the definition of the transformed T-tensor

$$
\bar{T}_{h i j k}=\bar{L}_{C_{h i j} \bar{l}_{k}}+\bar{C}_{h i j} \bar{l}_{k}+\bar{C}_{h i k} \bar{l}_{j}+\bar{C}_{h j k} \bar{l}_{i}+\bar{C}_{i j k} \bar{l}_{h} .
$$

The transformed T-tensor for some important special Finsler spaces can be deduced from the above result.

Corollary 3.2. Under a Kropina change, $\bar{L}=L^{2} / \beta ; L$ being Reimannian, the transformed T-tensor takes the form:

$$
\begin{aligned}
\bar{T}_{l i j k}= & \frac{2 \bar{L}}{L^{2} b^{2}}\left(h_{l i} h_{j k}+h_{l j} h_{i k}+h_{l k} h_{i j}\right)+\frac{2 \bar{L}^{2}}{\beta L^{2} b^{2}}\left(h_{l i} m_{j} m_{k}+h_{l j} m_{i} m_{k}+h_{i j} m_{l} m_{k}\right. \\
& \left.+h_{j k} m_{i} m_{l}+h_{l k} m_{i} m_{j}+h_{i k} m_{j} m_{l}\right)+\frac{6 \bar{L}^{3}}{\beta^{2} L^{2} b^{2}} m_{l} m_{i} m_{j} m_{k} .
\end{aligned}
$$


It is to be noted that the above result is also obtained by Shibata [18].

Corollary 3.3. Under a conformal change $\bar{L}=e^{\sigma} L$, the transformed T-tensor takes the form

$$
\bar{T}_{l i j k}=e^{3 \sigma} T_{l i j k}
$$

Corollary 3.4. Under a Randers change $\bar{L}=L+\beta, L$ being Riemannian, the $T$ tensor takes the form:

$$
\bar{T}_{l i j k}=-\frac{\Theta_{1}}{4 L^{3}}\left(h_{l i} h_{j k}+h_{l j} h_{i k}+h_{l k} h_{i j}\right)
$$

where $\Theta_{1}:=L^{2} b^{2}+\beta^{2}+2 L \beta$.

The above case has been studied by Matsumoto [11].

Corollary 3.5. Under a $\beta$-conformal change $\bar{L}=e^{\sigma} L+\beta$; L being Finslerian, the transformed T-tensor takes the form:

$$
\begin{aligned}
\bar{T}_{l i j k}= & \frac{e^{\sigma} \bar{L}^{2}}{L^{2}} T_{l i j k}-\frac{e^{\sigma} \Theta}{4 L^{3}}\left(h_{l i} h_{j k}+h_{l j} h_{i k}+h_{l k} h_{i j}\right) \\
& +\frac{e^{\sigma} \bar{L}}{2 L}\left(C_{l i j} m_{k}+C_{i j k} m_{l}+C_{j l k} m_{i}+C_{l i k} m_{j}\right) \\
& -\frac{e^{\sigma} \bar{L}}{2 L}\left(h_{i j} C_{l k \beta}+h_{j l} C_{i k \beta}+h_{i l} C_{j k \beta}+h_{l k} C_{i j \beta}+h_{j k} C_{i l \beta}+h_{i k} C_{j l \beta}\right),
\end{aligned}
$$

where $\Theta:=L^{2} b^{2}+\beta^{2}+2 e^{\sigma} L \beta$.

Corollary 3.6. Under a $\beta$-conformal change, a necessary condition for the vanishing of the transformed T-tensor is that

$$
T=\frac{\left(n^{2}-1\right) \Theta}{4 L \bar{L}^{2}}+\frac{(n-1) L}{\bar{L}} C_{\beta},
$$

where $T=g^{l j} g^{i k} T_{l i j k}$.

\section{The b-condition}

In this section we introduce and investigate what we call the b-condition. We study the effect of subjecting some special Finsler spaces to this condition. In the following we assume that we are given a generalized $\beta$-conformal change $\bar{L}=f\left(e^{\sigma} L, \beta\right)$ with $\beta=b_{i} y^{i}=b^{i} y_{i}$.

A Finsler manifold $(M, L)$ is said to satisfy the b-condition if

$$
b^{i} C_{i j k}=0 .
$$

Theorem 4.1. For $n>2$, the following two assertions are equivalent:

(a) The b-condition is invariant under a generalized $\beta$-conformal change. 
(b) The generalized $\beta$-conformal change is an energy $\beta$-change.

Proof.

(a) $\Rightarrow$ (b): Let $b^{i} C_{i j k}=0$. Then, $b^{i} \bar{C}_{i j k}=0$ and we have, by Proposition 1.3,

$$
e^{\sigma} p_{-1}\left(m^{2} h_{j k}+2 m_{j} m_{k}\right)+p_{02} m^{2} m_{j} m_{k}=0 .
$$

Contracting by $b^{j}$, we get $3 e^{\sigma} p_{-1}=-m^{2} p_{02}$. Hence,

$$
e^{\sigma} p_{-1}\left(m^{2} h_{j k}-m_{j} m_{k}\right)=0,
$$

contracting again by $g^{j k}$, we get

$$
(n-2) p_{-1}=0 .
$$

Since $n>2$, then $p_{-1}=0$ and hence the result follows from Theorem 2.1 .

(b) $\Rightarrow(\mathbf{a})$ : Let the generalized $\beta$-conformal change be an energy $\beta$-change. Then, by Theorem 2.1, we obtain $\bar{C}_{i j k}=e^{\sigma} p C_{i j k}$. Hence the result.

Theorem 4.2. Under a generalized Randers change, if $(M, L)$ satisfies the $b$-condition, the generalized Randers space $(M, \bar{L})$ can not satisfy the $b$-condition.

Proof. Let $(M, L)$ satisfy the b-condition $b^{i} C_{i j k}=0$. If $(M, \bar{L})$ satisfies the bcondition, then $b^{i} \bar{C}_{i j k}=0$, and consequently,

$$
\frac{1}{2 L} b^{i}\left(2 \bar{L} C_{i j k}+h_{i j} m_{k}+h_{j k} m_{i}+h_{i k} m_{j}\right)=0,
$$

or

$$
\frac{L}{2 \bar{L}^{2}}\left(m^{2} h_{j k}+2 m_{j} m_{k}\right)=0
$$

which, by contraction by $g^{j k}$, yields a contradiction: $n=-1$.

Theorem 4.3. Consider the generalized $\beta$-change (1.1). In each of the following cases

(a) two-dimensional Finsler space,

(b) three-dimensional Finsler space satisfying the condition $L(x,-y)=L(x, y)$,

(c) quasi-C-reducible space with $b^{i} b^{j} Q_{i j} \neq 0$,

(d) C-reducible space,

(e) The transformed space $(M, \bar{L})$ with L Riemannian,

if the given Finsler space $(M, L)$ satisfies the b-condition, then it is Riemannian.

Proof.

The proof of (a) and (b) runs on in a similar manner as given in [15] for a concurrent vector fields.

(c) Contracting (2.2) by $b^{i} b^{j}$, we get

$$
b^{i} b^{j} Q_{i j} C_{k}=0 .
$$


Hence, $C_{k}=0$ for $b^{i} b^{j} Q_{i j} \neq 0$.

(d) Contracting (2.4) by $b^{i} b^{j}$, we get

$$
m^{2} C_{k}=0 .
$$

Consequently, $C_{k}=0$.

(e) Let $(M, \bar{L})$ be a Finsler space with $(\alpha, \beta)$-metric, then

$$
\bar{C}_{i j k}=\frac{e^{\sigma} p_{-1}}{2}\left(h_{i j} m_{k}+h_{j k} m_{i}+h_{k i} m_{j}\right)+\frac{p_{02}}{2} m_{i} m_{j} m_{k} .
$$

The condition that $b^{i} \bar{C}_{i j k}=0$ leads to

$$
e^{\sigma} p_{-1}\left(m^{2} h_{j k}+2 m_{j} m_{k}\right)+p_{02} m^{2} m_{j} m_{k}=0 .
$$

Contracting by $b^{j}$, we get $3 e^{\sigma} p_{-1}=-m^{2} p_{02}$. Hence,

$$
e^{\sigma} p_{-1}\left(m^{2} h_{j k}-m_{j} m_{k}\right)=0,
$$

which, by contracting by $g^{j k}$, yields

$$
(n-2) p_{-1}=0 .
$$

Thus, if $n=2$, the result follows by (a) and if $p_{-1}=0$, then $p_{02}=0$ and hence $\bar{C}_{i j k}=0$.

Theorem 4.4. A semi-C-reducible Finsler space satisfying the b-condition is either Riemannian or C2-like.

Proof. Contracting (2.3) by $b^{j} b^{k}$, we have $r m^{2} C_{i}=0$. Since $m^{2} \neq 0$, then either $r=0$, which implies that the space is $C_{2}$-like, or $C_{i}=0$, which implies that the space is Riemannian.

Theorem 4.5. If an $S_{3}$-like Finsler space $(M, L)$ satisfies the $b$-condition, then its vertical curvature tensor $S_{\text {hijk }}$ vanishes.

Proof. Contracting (2.11) by $b^{l}$, we get

$$
S\left(h_{i k} m_{j}-h_{i j} m_{k}\right)=0 .
$$

Again, contracting (4.1) by $g^{i j}$, we have $(n-2) S m_{k}=0$. As $n>4$ and $m_{k} \neq 0$, it follows that $S=0$ and consequently, $S_{l i j k}=0$.

Lemma 4.6. If a Finsler space satisfies the $b$-condition, then we have $\left.b^{i}\right|_{h}=0$ and, consequently,

$$
\left.C_{i j h}\right|_{k} b^{h}=\left.C_{i j k}\right|_{h} b^{h}=0 .
$$

Proof. From the definition of vertical covariant derivative of Cartan connection, we have

$$
\left.b^{i}\right|_{h}=\dot{\partial}_{h} b^{i}+b^{m} C_{m h}^{i}=\dot{\partial}_{h}\left(b_{j} g^{i j}\right)=\left(\dot{\partial}_{h} b_{j}\right) g^{i j}+b_{j} \dot{\partial}_{h} g^{i j}=0
$$

and hence $\left.C_{i j k}\right|_{h} b^{h}=\left.C_{i j h}\right|_{k} b^{h}=\left.\left(C_{i j h} b^{h}\right)\right|_{k}=0$. 
It is well-known that if $(M, L)$ is Riemannian, then the T-tensor vanishes. But the converse is not true in general. The next result shows that the converse is true in the case where $(M, L)$ satisfies the b-condition.

Theorem 4.7. A Finsler space satisfying the b-condition is Riemannian if and only if the T-tensor $T_{h i j k}$ vanishes.

Proof. It is clear that if the space is Riemannian then the T-tensor vanishes. On the other hand, if the T-tensor vanishes, then

$$
\left.L C_{h i j}\right|_{k}+C_{h i j} l_{k}+C_{h i k} l_{j}+C_{h j k} l_{i}+C_{i j k} l_{h}=0 .
$$

Contracting by $b^{i}$, using Lemma 4.6, we have $\frac{\beta}{L} C_{h j k}=0$. Hence $C_{h j k}=0$.

Let us write

$$
T_{i j}:=T_{i j h k} g^{h k}=\left.L C_{i}\right|_{j}+l_{i} C_{j}+l_{j} C_{i} .
$$

By contracting (4.2) by $b^{i}$, making use of Lemma 4.8, we have

$$
T_{i j} b^{i}=(\beta / L) C_{j}
$$

Hence, we have

Corollary 4.8. A Finsler space satisfying the b-condition is Riemannian if and only if the tensor $T_{i j}$ vanishes.

\section{Projective change and generalized $\beta$-conformal change}

In this section we will be guided by Matsomoto [13] and Shibata [18]. For two Finsler spaces $(M, L)$ and $(M, \bar{L})$ with the same underlying manifold $M$, if every geodesic on of $(M, L)$ is also a geodesic of $(M, \bar{L})$ and vice versa, the change $L \longrightarrow \bar{L}$ of Finsler metrics is said to be projective. A geodesic on $(M, L)$ is characterized by

$$
\frac{d y^{i}}{d t}+2 G^{i}=w y^{i}, \frac{d x^{i}}{d t}=y^{i}
$$

where $w=\left(d^{2} s / d t^{2}\right) /(d s / d t)^{2}$ and $G^{i}(x, y)=\frac{1}{2} \gamma_{j k}^{i} y^{j} y^{k}$ is the canonical spray of $(M, L)$. We are going to find out a condition for a generalized $\beta$-conformal change to be projective.

Consider the left hand side of Euler-Lagrange equations

$$
\mathcal{E}_{i}:=\partial_{i} L-\frac{d}{d t}\left(\dot{\partial}_{i} L\right)
$$

Proposition 5.1. Under a generalized $\beta$-conformal change $\bar{L}=f\left(e^{\sigma} L, \beta\right)$, the functions (5.1) are transformed according to

$$
f \overline{\mathcal{E}}_{i}=L e^{\sigma} p \mathcal{E}_{i}+L q_{0} m_{i} m^{r} \mathcal{E}_{r}+\varphi_{i},
$$

where

$$
\begin{gathered}
\varphi_{i}:=L^{2} e^{\sigma} p \sigma_{i}-\left(p L e^{\sigma} l_{i}-q_{0} \beta m_{i}\right) \sigma_{0}+2 q F_{0 i}-q_{0} E_{00} m_{i} \\
\sigma_{i}:=\partial_{i} \sigma, \quad F_{i j}:=\frac{1}{2}\left(b_{i \mid j}-b_{j \mid i}\right), \quad E_{i j}:=\frac{1}{2}\left(b_{i \mid j}+b_{j \mid i}\right), \\
\sigma_{0}=\sigma_{i} y^{i}, \quad F_{0 i}=F_{l i} y^{l}, \quad E_{00}=E_{i j} y^{i} y^{j}
\end{gathered}
$$


Proof. Making use of the homogeneity of $f, \overline{E_{i}}$ can be computed as follows.

$$
\begin{aligned}
\overline{\mathcal{E}_{i}}= & \partial_{i} f-\frac{d}{d t}\left(\dot{\partial}_{i} f\right) \\
= & f_{1}\left(\sigma_{i} e^{\sigma} L+e^{\sigma} \partial_{i} L\right)+f_{2}\left(N_{i}^{r} b_{r}+b_{j \mid i} y^{j}\right)-\frac{d}{d t}\left(e^{\sigma} f_{1} l_{i}+f_{2} b_{i}\right) \\
= & f_{1} \sigma_{i} e^{\sigma} L+f_{1} e^{\sigma} \partial_{i} L+f_{2} N_{i}^{r} b_{r}+f_{2} b_{j \mid i} y^{j}-f_{1} l_{i} e^{\sigma} \sigma_{r} y^{r}-f_{1} e^{\sigma} \frac{d l_{i}}{d t}-e^{\sigma} l_{i} \frac{d f_{1}}{d t} \\
& -b_{i} \frac{d f_{2}}{d t}-f_{2}\left(b_{i \mid j} y^{j}+N_{i}^{r} b_{r}\right) \\
= & f_{1} e^{\sigma} \mathcal{E}_{i}+f_{1} L \sigma_{i} e^{\sigma}-f_{1} \sigma_{0} e^{\sigma} l_{i}+2 f_{2} F_{0 i}-\frac{d f_{2}}{d t} m_{i} .
\end{aligned}
$$

Using the relation $\frac{d y_{r}}{d t}=y^{s} \partial_{s} y_{r}+g_{r s} \frac{d y^{s}}{d t}$, the last term $\frac{d f_{2}}{d t}$ of (5.4) is given by

$$
\begin{aligned}
\frac{d f_{2}}{d t} & =f_{21} \frac{d \tilde{L}}{d t}+f_{22} \frac{d \beta}{d t} \\
& =-\frac{\beta f_{22}}{e^{\sigma} L}\left(e^{\sigma} y^{r} \partial_{r} L+e^{\sigma} l_{r} \frac{d y^{r}}{d t}+L \frac{d e^{\sigma}}{d t}\right)+f_{22}\left(E_{00}+N_{s}^{r} b_{r} y^{s}+b_{r} \frac{d y^{r}}{d t}\right) \\
& =f_{22} E_{00}-L f_{22} m_{i} m^{r} \mathcal{E}_{r}-\beta f_{22} \sigma_{0} .
\end{aligned}
$$

Now, substituting (5.5) into (5.4), we get

$$
\begin{aligned}
f \overline{\mathcal{E}_{i}} & =L e^{\sigma} p \mathcal{E}_{i}+q_{0} L m_{i} m^{r} \mathcal{E}_{r}+L^{2} e^{\sigma} p \sigma_{i}-\left(p L e^{\sigma} l_{i}-q_{0} \beta m_{i}\right) \sigma_{0}+2 q F_{0 i}-q_{0} E_{00} m_{i} \\
& =L p e^{\sigma} \mathcal{E}_{i}+q_{0} L m_{i} m^{r} \mathcal{E}_{r}+\varphi_{i} .
\end{aligned}
$$

Theorem 5.2. A generalized $\beta$-conformal change is projective if and only if the vector $\varphi_{i}$ vanishes.

Proof. Let the generalized $\beta$-conformal change be projective. Then, $\mathcal{E}_{i}=0$ is equivalent to $\overline{\mathcal{E}}_{i}=0$ and consequently, $\varphi_{i}=0$ by (5.2).

Conversely, if $\varphi_{i}=0$, then (15.2) shows that $\mathcal{E}_{i}=0$ implies $\overline{\mathcal{E}}_{i}=0$. On the other hand, if $\overline{\mathcal{E}}_{i}=0$ and $\varphi_{i}=0$, then $e^{\sigma} p \mathcal{E}_{i}+q_{0} m_{i} m^{r} \mathcal{E}_{r}=0$. Contracting the last equation by $m^{i}$, taking into account that $e^{\sigma} p+m^{2} q_{0} \neq 0$, we get $\mathcal{E}_{r} m^{r}=0$. Consequently, $\mathcal{E}_{i}=0$.

From the above theorem, we retrieve the following two results due to Shibata [19] and Hashiguchi and Ichijo [5] respectively.

Corollary 5.3. A $\beta$-change is projective if and only if $2 q F_{0 i}=q_{0} E_{00} m_{i}$.

Corollary 5.4. A Randers change is projective if and only if $F_{0 i}=0$, that is, $b_{i}$ is gradient.

The following two results are a generalized version of Shibata's result [19] and Matsumoto's result [13].

Theorem 5.5. Assume that the generalized $\beta$-conformal change (1.1) is projective and $L$ is Minkowskian, then the Weyl torsion $\bar{W}_{i j}^{h}$ and the Douglas tensor $\bar{D}_{i j k}^{h}$ of $(M, \bar{L})$ vanish. Consequently, $(M, L)$ with dim $M>2$ is projectively flat. 
Proof. The Weyl torsion tensor is given by [13]:

$$
W_{i j}^{h}=\stackrel{\circ}{R}_{i j}^{h}+\frac{1}{n+1} \mathfrak{A}_{(i, j)}\left\{y^{h} \stackrel{\circ}{R}_{i j}+\delta_{i}^{h} \stackrel{\circ}{R}_{j}\right\},
$$

where $\stackrel{\circ}{R}_{i j}=\stackrel{\circ}{R}_{i j h}^{h}, \stackrel{\circ}{R}_{j}=\frac{1}{n+1}\left(n \stackrel{\circ}{R}_{0 j}+\stackrel{\circ}{R}_{j 0}\right)$ and $\stackrel{\circ}{R}_{i j k}^{h}$ is the h-curvature of the Berwald connection. Since $(M, L)$ is Minkowskian, then $\stackrel{\circ}{R}_{i j k}^{h}=0$, and so $\stackrel{\circ}{R}_{i j}=\stackrel{\circ}{R}_{i}=0$. Consequently, $W_{i j}^{h}=0$. By the invariance of $W_{i j}^{h}$ under a projective change, we have $\bar{W}_{i j}^{h}=0$.

The Douglas tensor is given by [13]:

$$
D_{i j k}^{h}=\stackrel{\circ}{P}_{i j k}^{h}+\frac{1}{n+1}\left(y^{h} \stackrel{\circ}{P}_{i j} \stackrel{\circ}{\mid}_{k}+\mathfrak{S}_{(i, j, k)}\left\{\delta_{i}^{h} \stackrel{\circ}{P}_{j k}\right\}\right),
$$

where $\stackrel{\circ}{P}_{i j}=\stackrel{\circ}{P}_{i j h}^{h}, \stackrel{\circ}{P}_{i j k}^{h}$ is the hv-curvature of the Berwald connection and $\stackrel{\circ}{\mid}$ denotes the vertical covariant derivative with respect to the Berwald connection $G_{i j}^{h}$. Since $(M, L)$ is Minkowskian, then $\stackrel{\circ}{P}_{i j k}^{h}=0$, and so $\stackrel{\circ}{P}_{i j}=0$. Consequently, $D_{i j k}^{h}=0$. By the invariance of $D_{i j k}^{h}$ under a projective change, we have $\bar{D}_{i j k}^{h}=0$.

Finally, as $W_{i j}^{h}=0, D_{i j k}^{h}=0$ and $\operatorname{dim} M>2,(M, L)$ is thus projectively flat [13].

Theorem 5.6. Assume that the generalized $\beta$-conformal change is projective and $L$ is Riemannian, then the projective hv-curvature tensor $\bar{D}_{i j k}^{h}$ of $(M, \bar{L})$ vanishes.

Proof. Since $(M, L)$ is Riemannian, then $\stackrel{\circ}{P}_{i j k}^{h}=0$, and $\stackrel{\circ}{P}_{i j}=0$. Consequently, $D_{i j k}^{h}=0$. By the invariance of $D_{i j k}^{h}$ under a projective change, we have $\bar{D}_{i j k}^{h}=0$.

Theorem 5.7. If $\varphi_{i}=0$, then $(M, \bar{L})$ is of scalar curvature if and only if $(M, L)$ is of scalar curvature.

Proof. According to Szabó [21], a Finsler space is of scalar curvature if and only if $W_{i j}^{h}=0$ vanishes identically. Let $\varphi_{i}=0$, then by Theorem 5.2 the generalized $\beta$ conformal change is projective. Now, let $(M, L)$ be of scalar curvature, then $W_{i j}^{h}=0$. But $\bar{W}_{i j}^{h}=W_{i j}^{h}$, hence, $\bar{W}_{i j}^{h}=0$. Consequently, $(M, \bar{L})$ is of scalar curvature. Conversely, let $(M, \bar{L})$ be of scalar curvature, then $\bar{W}_{i j}^{h}=0$ which leads to $W_{i j}^{h}=0$, hence, $(M, L)$ is of scalar curvature.

In the Riemannian case the term "of scalar curvature" reduces to the term "of constant curvature". Thus, we generalize Yasuda and Shimada's result [24].

Corollary 5.8. Under a generalized $\beta$-conformal change, if $\varphi_{i}=0$ and $(M, L)$ is Riemannian, then the Finsler space $(M, \bar{L})$ is of scalar curvature if and only if $(M, L)$ is of constant curvature.

\section{References}

[1] S. H. Abed, Conformal $\beta$-changes in Finsler spaces, Proc. Math. Phys. Soc. Egypt, 86 (2008), 79-89. ArXiv No.: math.DG/0602404. 
[2] S. H. Abed, Cartan connections associated with a $\beta$-conformal change in Finsler geometry, Tensor, N. S., 70 (2008), 146-158. ArXiv No.: math.DG/0701491.

[3] M. A. Eliopoulos, A generalized metric space for electromagnetic theory, Acad. Roy. Belg. Bull. CI. Sci., (5) 50 (1965), 986-995.

[4] M. Hashiguchi, On conformal transformations of Finsler metrics, J. Math. Kyoto Univ., 16 (1976), 25-50.

[5] M. Hashiguchi and Y. Ichijo, Randers spaces with rectilinear geodesic, Rep. Fac. Sci. Kagoshima Univ. (Math. Phys. Chem.), 13 (1980), 33-40.

[6] R. S. Ingarden, On the geometrically absolute representation in the electron microscope, Trav. Soc. Lett. Wroclaw, B 45 (1957), 60p.

[7] H. Izumi, Conformal transformations of Finsler spaces, Tensor, N. S., 31 (1977), $33-41$.

[8] V. K. Kropina, On Projective two-dimentional Finsler spaces with special metric, Truday Sem. Vektor Tenzor Anal., 11 (1961), 277-292.

[9] M. Matsumoto, On C-reducible Finsler spaces, Tensor, N. S., 24 (1972), 29-37.

[10] M. Matsumoto, V-transformations of Finsler spaces. I. Definition, infinitesimal transformations and isometries, J. Math. Kyoto Univ., 12 (1972), 479-512.

[11] M. Matsumoto, On Finsler spaces with Randers metric and special forms of important tensors, J. Math. Kyoto Univ., 14 (1974), 477-498.

[12] M. Matsumoto, On three dimensional Finsler spaces satisfying the $T$ and $B^{p}$ conditions, Tensor, N. S., 29 (1975), 13-20.

[13] M. Matsumoto, Projective changes of Finsler metrics and projectively flat Finsler spaces, Tensor, N. S., 34 (1980), 303-315.

[14] M. Matsumoto, Foundations of Finsler geometry and special Finsler spaces, Kaiseisha Press, Otsu, Japan, 1986.

[15] M. Matsumoto and K. Eguchi, Finsler spaces admitting a concurrent vector field, Tensor, N. S., 28 (1974), 239-249.

[16] M. Matsumoto and C. Shibata, On semi-C-reducibility, T-tensor $=0$ and $S_{4}$ likeness of Finsler spaces, J. Math. Kyoto Univ., 19 (1979), 301-314.

[17] G. Randers, On an asymmetrical metric in the four-space of general relativity, Phys. Rev., (2) 59 (1941), 195-199.

[18] C. Shibata, On Finsler spaces with Korpina metric, Rep. Math. Phys., 13 (1978), 117-128

[19] C. Shibata, On invariant tensors of $\beta$-changes of Finsler metrics, J. Math. Kyoto Univ., 24 (1984), 163-188. 
[20] C. Shibata, H. Shimada, M. Azuma and H. Yasuda, On Finsler space with Randers metric, Tensor, N. S., 31 (1977), 219-226.

[21] Z. I. Szabò, Ein Finslerschen raum ist gerade dann von skalarer krümmung, wenn seine Weylsche projektivkrümmung verschwindet, Acta Sci. Math. Szeged, 39 (1977), 163-168.

[22] A. A. Tamim and Nabil L. Youssef, On generalized Randers manifolds, Algebras, Groups and Geometries, 16 (1999), 115-126.

[23] A. Tayebi and E. Peyghan On Ricci-tensor of Randers metrics, To appear in Journal of Geometry and Physics, 2010. ArXiv Number:1005.5590v1 [math.DG].

[24] H. Yasuda and H. Shimada, On Randers spaces of scalar curvature, Rep. Math. Phys., 11 (1977), 347-360.

[25] Nabil L. Youssef, S. H. Abed and S. G. Elgendi, Generalized $\beta$-conformal change of Finsler metrics, To appear in "Int. J. Geom. Meth. Mod. Phys.", 7, 4 (2010). ArXiv Number: 0906.5369 [math.DG].

[26] Nabil L. Youssef, S. H. Abed and A. Soleiman, A global theory of conformal Finsler geometry, Tensor, N. S., 69 (2008), 155-178. ArXiv Number: math.DG/0610052

[27] Nabil L. Youssef, S. H. Abed and A. Soleiman, A global approach to the theory of special Finsler manifolds, J. Math. Kyoto Univ., 48, 4 (2008), 857-893. ArXiv Number: 0704.0053 [math.DG].

[28] Nabil L. Youssef, S. H. Abed and A. Soleiman, Concurrent $\pi$-vector fields and eneregy $\beta$-change, Int. J. Geom. Meth. Mod. Phys., 6 (2009), 1003-1031. ArXiv Number: 0805.2599 [math.DG]. 